\title{
MD Study of Functionalized Single-Walled Carbon Nanotube*
}

\author{
Shuhei INOUE** and Yukihiko MATSUMURA** \\ ${ }^{* *}$ Energy and Environmental Engineering Division, Faculty of Engineering, Hiroshima University \\ 1-4-1 Kagamiyama, Higashi-Hiroshima, Hiroshima 739-8527, Japan \\ E-mail: shu18@hiroshima-u.ac.jp
}

\begin{abstract}
Carbon nanotubes possess outstanding properties such as high thermal conductivity, ultra-high physical strength, and durability. With regard to applications, a single-walled carbon nanotube (SWCNT) is a great candidate for a new material. Furthermore, a metal-coated SWCNT has been experimentally developed and its properties have attracted much attention. In this study, we simulated the deposition process of metal species onto an SWCNT to estimate its resulting physical strength and thermal diffusivity using a classical molecular dynamics method. Consequently, we found that the physical strength of the metal-coated SWCNT was similar to that of an uncoated SWCNT, whereas its thermal diffusivity showed a decrease of $90 \%$.
\end{abstract}

Key words: Heat Transfer, Metal Deposition, Physical Strength, Molecular Dynamics Method

\section{Introduction}

Originally, the structure of a carbon nanotube ${ }^{(1)}$ was regarded as merely a unique carbon molecule or crystal. As research progressed, a single-walled carbon nanotube (SWCNT) ${ }^{(2)}$ became one of the most attractive new materials, because of its outstanding physical properties ${ }^{(3-8)}$. With regard to the applications of an SWCNT, its affinity for metal species is a key issue. First, a chemically associated SWCNT was reported ${ }^{(9,10)}$. More recently, Duong et al. ${ }^{(11)}$ experimentally attempted to deposit a metal on a vertically aligned SWCNT surface and then discussed the interactions. Zhang et al. ${ }^{(12)}$ coated an isolated SWCNT with some metal species. Both of these papers indicated that the interaction between SWCNTs and metals affects the behavior of the deposition processes. Some metal species exhibit quite good affinity, but the others are deposited on the SWCNT surface to form granular structures. We simulated this phenomenon by using classical molecular dynamics, and the results showed good agreement with the experiments. However, the properties of a metal-coated SWCNT (i.e., a functionalized SWCNT) are among the most important pieces of information for engineering applications. In this study, therefore, we inferred the physical strength and thermal diffusivity of a functionalized SWCNT. We found that the physical strength of the metal-coated SWCNT was not so different from that of the uncoated SWCNT, whereas its thermal diffusivity showed a decrease of $90 \%$.

\section{Methodology}

\subsection{Deriving parameters for many-body potential function}

The Brenner-Tersoff potential ${ }^{(13)}$ was applied to the carbon-carbon interaction, and the classical many-body potential was applied to the metal-metal and carbon-metal interactions as a function of the bond number. With respect to the metal-metal potential function, the Finnis-Sinclair (FS) ${ }^{(14)}$ potential is generally recognized as one of the most acceptable,

${ }^{*}$ Received 15 Feb., 2011 (No. 11-0110) [DOI: 10.1299/jtst.6.256]

Copyright $@ 2011$ by JSME 
Table 1. Potential parameters for MD simulations

\begin{tabular}{|c|c|c|c|c|c|c|c|c|c|}
\hline atom & $S$ & $\begin{array}{c}\beta \\
\left(\AA^{-1}\right)\end{array}$ & $\begin{array}{c}D_{e l} \\
(\mathrm{eV})\end{array}$ & $\begin{array}{c}D_{e 2} \\
(\mathrm{eV})\end{array}$ & $C_{D}$ & $\begin{array}{l}R_{e l} \\
(\AA)\end{array}$ & $\begin{array}{l}R_{e 2} \\
(\AA)\end{array}$ & $C_{R}$ & $\begin{array}{cc}R_{1} & R_{2} \\
(\AA) & (\AA)\end{array}$ \\
\hline $\mathrm{Ti}-\mathrm{Ti}$ & 1.3 & 2.331 & 0.6500 & 0.2001 & 0.3700 & 3.819 & 2.411 & 0.2357 & 2.7 \\
\hline $\mathrm{Au}-\mathrm{Au}$ & 1.3 & 1.750 & 0.5290 & 1.3510 & 1.5251 & 3.3043 & 0.7573 & 0.2939 & 2.9 \\
\hline atom & \multicolumn{2}{|c|}{$D e(\mathrm{eV})$} & $S$ & $\beta\left(\AA^{-1}\right)$ & $R_{e}(\AA)$ & $R_{l}(\AA)$ & $R_{2}(\AA)$ & $b$ & $\delta$ \\
\hline $\mathrm{Ti}-\mathrm{C}$ & \multicolumn{2}{|c|}{3.6240} & 1.3 & 1.466 & 1.8000 & 2.7 & 3.0 & 0.0600 & -0.5000 \\
\hline $\mathrm{Au}-\mathrm{C}$ & \multicolumn{2}{|c|}{2.1840} & 1.3 & 2.0745 & 1.9030 & 2.9 & 3.3 & 0.0970 & -0.5350 \\
\hline
\end{tabular}
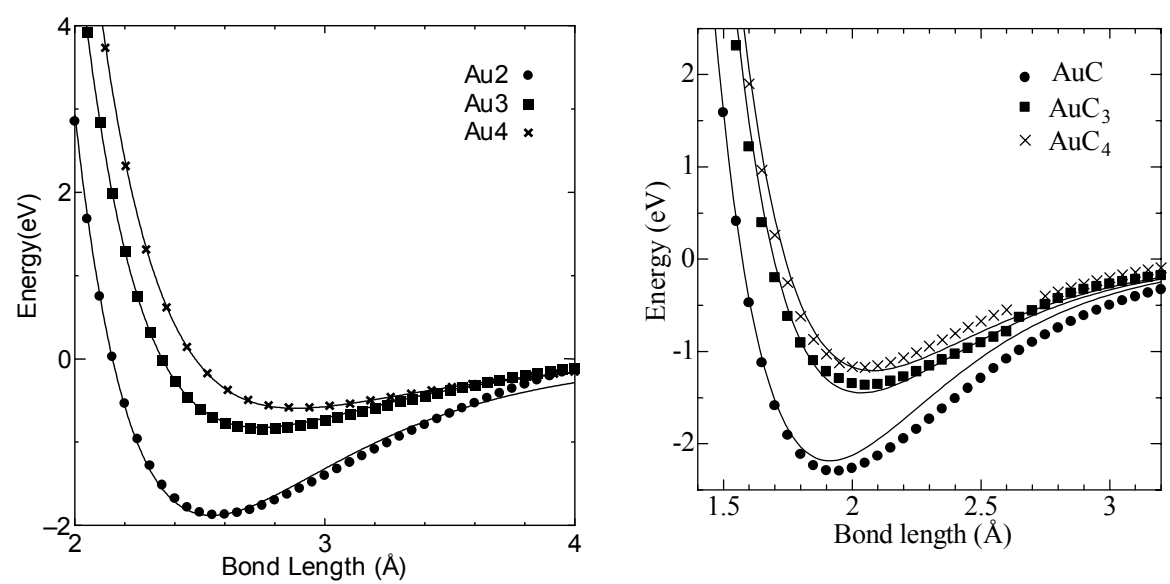

Fig. 1. Fitted potential curves from the lowest ground state derived by DFT calculation (Gaussian03, B3LYP/LANL2DZ). Dots show the binding energies, and the fitted curves are the bond order potential curves. In each case, various spin states are examined and their minimum values are employed.

especially for expressing fcc-bcc transitions. However, at present there is no promising function for the metal-carbon potential. Thus, as a last resort, the Lennard-Jones (LJ) potential is applied to express the metal-carbon interaction, even though the LJ potential was originally intended to express the monoatomic molecule behavior, and therefore, it cannot express the effect of the coordination number. Because the many-body potential is quite a reasonable choice to express the effect of the coordination number, we applied this potential to the metal-carbon interaction. Nevertheless, we had concerns about the mismatch between the depths of the potential functions when the FS potential was applied for the metal-metal interaction. Although the FS potential can express fcc-bcc transitions, the absolute potential depth has no affect on the transition, but only the relative depth affects the FS potential itself. Therefore, we also applied the many-body potential to the metal-carbon interaction. The parameter sets for the nickel-nickel, carbon-nickel, iron-iron, and carbon-iron interactions were based on the results of Shibuta and Maruyama ${ }^{(15)}$. However, the parameter sets for the gold-gold, carbon-gold, titanium-titanium, and carbon-titanium interactions were obtained by density functional theory (DFT) calculations. Gaussian 03 and Becke's three-parameter exchange functional with the Lee-Yang-Parr correlation functional (B3LYP) ${ }^{(16,17)}$ were used in this study. Moreover, the Los Alamos effective core potential plus DZ (LANL2DZ) ${ }^{(18)}$ was used as the basis set.

Because the method for deriving parameters was similar to that described in the abovementioned paper, the derived parameters are considered reliable and are explained here briefly. First, we assume that the symmetrical structure of Aux and AuCx clusters means that we need not take into account the Jahn-Teller ${ }^{(19)}$ distortion to calculate their total energy. The binding energy is calculated by subtracting the isolated energies from the total 
energy and then dividing the result by the number of bonds, as follows:

$$
\text { De }=\left(E_{\text {min. }}^{\text {total }}-E_{\text {metal,min. }}^{\text {isolat }}-N_{c} \cdot E_{\text {carbon,min. }}^{\text {isolated }}\right) / N_{b},
$$

where $E_{\text {min. }}^{\text {total }}$ is the total energy in a certain spin state, $E_{\text {metal,min. }}^{\text {isolat }}$ is the isolated energy

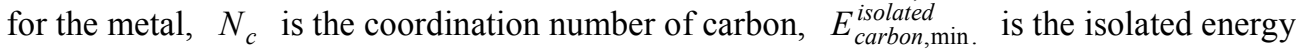
for carbon, and $N_{b}$ is the number of bonds. In each case, several spin states are calculated, and their minimum values corresponding to some distance are employed in the fitting. Although the clusters might take various spin states, we employ the spin state with the minimum energy. Figure 1 shows the binding energies of gold-gold and carbon-gold, as derived from the DFT calculation and fitted by the following bond order potential functions:

$E=V_{R}-V_{A}$

$V_{R}=\frac{D_{e}}{S-1} \exp \left\{-\beta \sqrt{2 S}\left(r-R_{e}\right)\right\}$

$V_{A}=B^{*} \frac{D_{e} S}{S-1} \exp \left\{1+b\left(N_{i}^{C}-1\right)\right\}^{\delta}$

$D_{e}=D_{e 1}+D_{e 2} \exp \left\{-C_{D}\left(N_{i}^{M}-1\right)\right\}$

$R_{e}=R_{e 1}-R_{e 2} \exp \left\{-C_{R}\left(N_{i}^{M}-1\right)\right\}$

$f(r)= \begin{cases}1 & \left(r<R_{1}\right) \\ 0.5 \cdot\left(1+\cos \frac{r-R_{1}}{R_{2}-R_{1}} \pi\right) & \left(R_{1}<r<R_{2}\right) \\ 0 & \left(R_{2}<r\right)\end{cases}$

$N_{i}^{C}=1+\sum_{\text {carbon }} f\left(r_{i k}\right)$

$N_{i}^{M}=1+\sum_{\text {metal }} f\left(r_{i k}\right)$

where $r$ denotes the distance between two atoms; $V_{R}$ and $V_{A}$ are the Morse-type potential $1^{(20)}$ repulsion and attraction terms, respectively; $S$ is the ratio of the effective repulsion and attraction terms; $R_{e}$ is the equilibrium bond length; $D_{e}$ is the potential

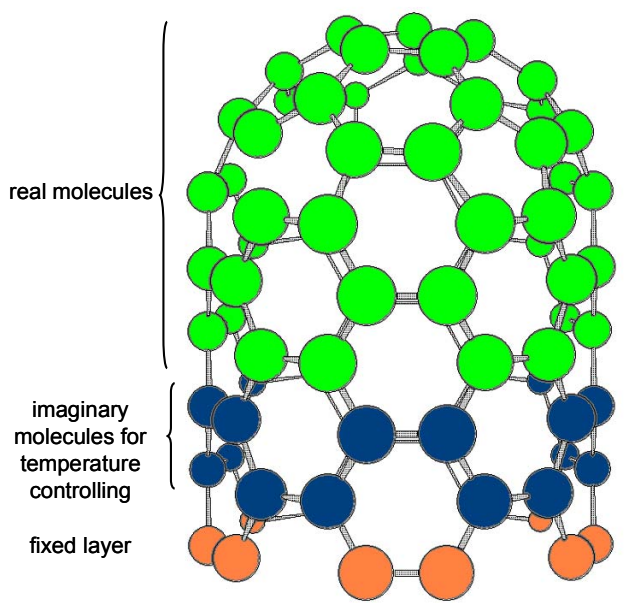

Fig. 2. Surface consisting of a capped carbon nanotube. The calculated system consists of 6 $\times 6$ units (2880 atoms) and has dimensions of $60 \AA \times 52 \AA \times 60 \AA$. 
depth (at $r=R_{e}$ ); and $N_{i}^{C}$ and $N_{i}^{M}$ are the coordination numbers, which are derived using the cut-off function $f(r)$. All these parameters are listed in Table 1.

\subsection{Expressing physical vapor deposition on vertically aligned SWCNT}

The vertically aligned single-walled carbon nanotube (VA-SWCNT) surface is represented by a capped short carbon nanotube with a $(5,5)$ chirality, as shown in Fig. 2 . In this study, the calculated system consists of $6 \times 6$ units with dimensions of $60 \AA \times 52 \AA \times 60 \AA$ and whose configuration resembles the fcc lattice (111). Atoms interact with each other within their cut-off distances. The first layer is a fixed layer, whereas the second and third layers are temperature-controlling layers that maintain their temperature by velocity scaling. The system has periodic boundary conditions in each direction, and the velocity Verlet algorithm is employed. Assuming a realistic metal evaporation in a chamber, the physical vapor deposition (PVD) on a VA-SWCNT surface is modeled as successive collisions by $\mathrm{M}_{13}$ clusters with small evaporation energy of $10.0 \mathrm{meV}$ each. The kinetic energy is thus about ten times less than the translational energy of a molecule at $1000 \mathrm{~K}$ in order to avoid the formation of clusters during the deposition. This is because the density of metal clusters is much higher than under experimental conditions, which are difficult to simulate owing to the calculation cost. However, even in comparison with the experimental condition, the size of the $\mathrm{M}_{13}$ clusters is reasonable. In this simulation, again owing to the calculation cost, the cluster size is maximized. The value was estimated from a PVD experiment, but further numerical experiments might be desirable. Each metal cluster is fully annealed above its melting point ( $\mathrm{Ni}, 1400 \mathrm{~K}$; $\mathrm{Au}, 1000 \mathrm{~K}$ ) and set as shown in Fig. 3. The VA-SWCNT is also annealed at $300 \mathrm{~K}$. These temperatures are lower than the bulk melting point expressed by the bond order potentials; however, the $\mathrm{M}_{13}$ cluster is very small, and therefore, its melting temperature is lower than that of the bulk (Ni, $1728 \mathrm{~K}$; Au, $1338 \mathrm{~K})$. Because the melting temperature of small clusters should be less than the bulk value, the potential parameters used in this simulation are correct in that sense. Nevertheless, application of the quantum molecular dynamics method is required to express the kinetics of the chemical reaction.

\subsection{Metal coating}

The dimensions of the calculated system are $60 \AA \times 100 \AA \times 100 \AA$, and a periodic boundary condition is applied in each direction. As shown in Fig. 4, an isolated SWCNT is $60 \AA$ in length with $(5,5)$ chirality. Both its ends are fixed, and its next two layers work to control its temperature. Before coating it with a metal, this carbon nanotube is annealed at $300 \mathrm{~K}$ by the velocity scaling technique. The molecules of the coating metal are modeled as

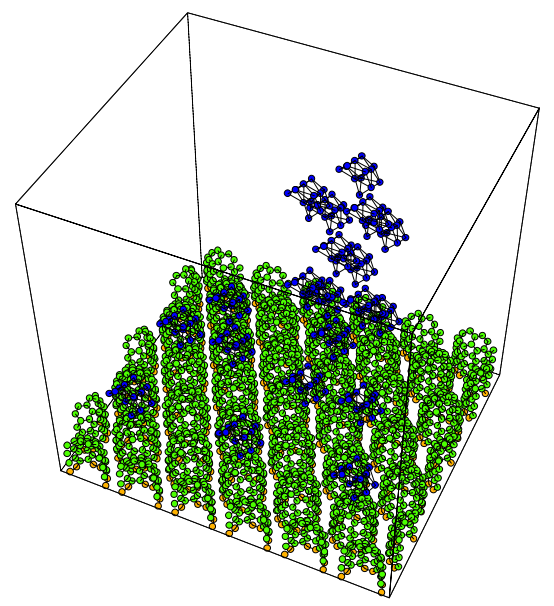

Fig. 3. Cluster configuration onto the flat CNT surface. The center of gravity of each cluster is above the center of the carbon nanotube or its hollow site. To express continuous deposition, some clusters are placed at distances of $10 \AA$ vertically. 
$\mathrm{M}_{13}$ clusters. This is representative of actual experiments, because in the conventional metal evaporation process, the average metal particle is roughly a few angstroms in diameter. The metal clusters are also fully annealed at 1200-1400 K, which clearly is higher than their melting temperature but lower than their boiling temperature. Based on the potential parameters, the melting temperature in the bulk form is approximately 1800-2000 K. However, in this study, the metal clusters are too small to exhibit bulk properties. In order to achieve a continuous metal evaporation coating, the fully annealed metal clusters are distributed at appropriate distances (15-20 $\AA$ ) and collided with the carbon nanotube at low evaporation energies of $10 \mathrm{meV}$ or $30 \mathrm{meV}$. This deposition energy is comparable to the experimental condition, because in the e-beam experiment, the electrons are accelerated by 5-10 keV.

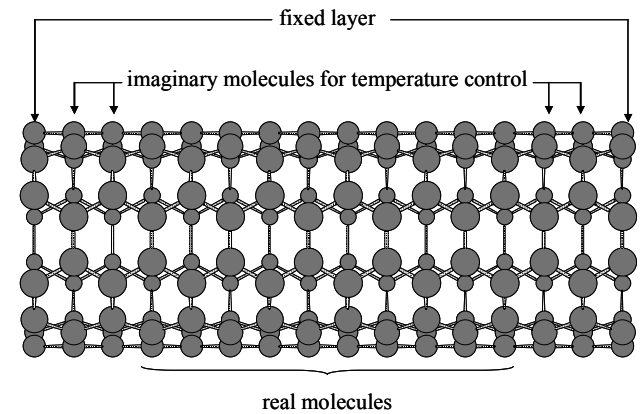

Fig. 4. Isolated single-walled carbon nanotube. The both ends are fixed layer and following two layers work to control the temperature.
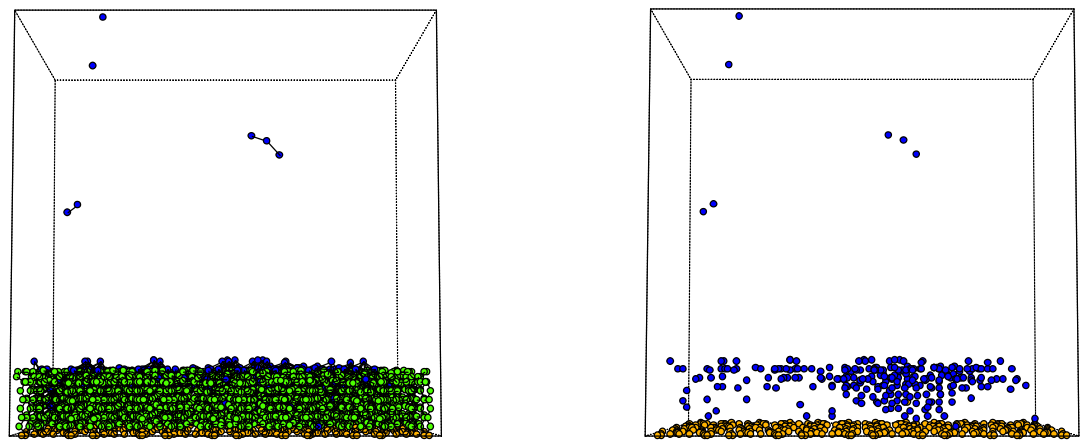

(a) Deposition of nickel clusters.
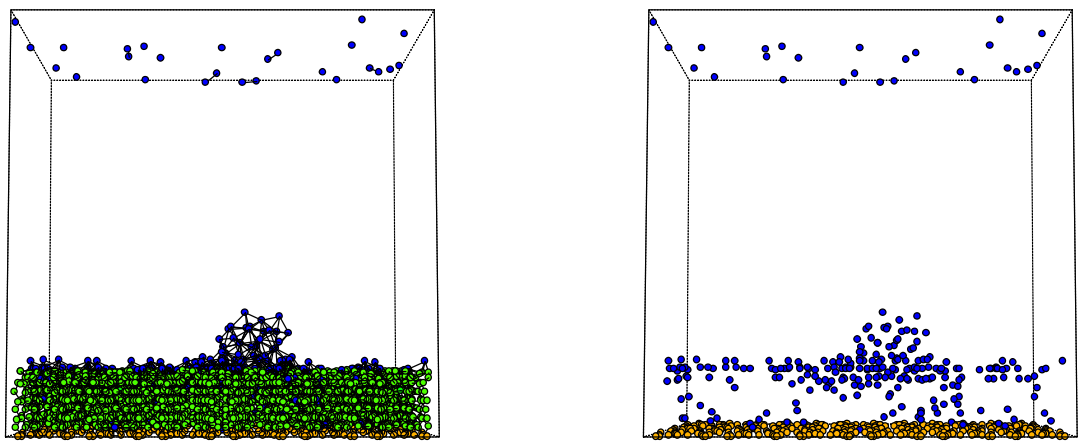

(b) Deposition of gold clusters.

Fig. 5. Metal PVD onto VA-SCNT surface. In both cases, small metal clusters collide with each other before arriving at the VA-SWCNT surface and form large clusters. The carbon atoms except for those in the fixed layer are hidden in the third figure to identify the metal 


\section{Results and discussion}

\subsection{Physical vapor deposition}

The initial configuration of the metal clusters is described above and shown in Fig. 3. Each cluster is placed immediately above the carbon nanotube, or its hollow site, to implement various collision models. In order to model continuous deposition, the clusters are placed at intervals of $10 \AA$. Figure 5 shows the result of PVD on a VA-SWCNT surface. In both cases, some of the metal clusters collided with each other to form a larger cluster before reaching the surface, while others arrived without clustering. In the case of nickel deposition, the deposited nickel atoms spread over the VA-SWCNT and formed a smooth surface, irrespective of earlier clustering. However, in the case of gold deposition, the smaller clusters of gold were able to spread over the VA-SWCNT surface, but the larger clusters were unable to spread and instead formed a granular structure. Comparing the potential curves of these metals, the equilibrium bond length appears slightly different, but the binding energy appears nearly identical. The potential depth at which they differ most is that at which their coordination number is unity. The potential depth of gold suddenly decreases as the coordination number increases. This may explain the formation of the granular structure, because the contact angle is closely related to wettability, which is well explained by solid-liquid affinity $\left(\varepsilon_{\mathrm{s}} / \varepsilon_{\mathrm{l}}\right)^{(21)}$. According to their results, the contact angle tends to increase as the solid-liquid affinity decreases. In the condensed phase the coordination number should be larger, so the affinity of gold should decrease in comparison to that of nickel. The electron configuration of an isolated gold atom is $[4 f 14,5 d 10,6 s 1]$ and that of a nickel atom is $[3 d 8,4 s 2]$. Therefore, the number of electrons that greatly contribute to binding should presumably be one for gold but more than two for nickel. The order of energy levels is $6 s, 4 f$, and $5 d$, but there is not much separation and sometimes this order can be reversed by forming a closed shell. In the case of gold, the $4 f$ orbital and $5 d$ orbital are closed shells already; therefore, only a single electron is unpaired. Thus, the gold atoms have a strong binding energy in the form of a dimmer. Conversely, the nickel atoms have some free electrons; therefore, their binding energy is less than that of the gold atoms. Compared to nickel deposition, gold deposition may require more stringent conditions to produce a flat surface. However, this can be achieved by carrying out deposition under a high vacuum condition and with a low deposition rate. Because only small clusters of gold can spread over the VA-SWCNT surface, it is essential to avoid clustering that occurs by colliding before reaching the surface.

\subsection{Metal coating on SWCNT}

Figure 6 shows the simulation results of continuous metal evaporation coating for (a) Ti, (b) $\mathrm{Fe}$, (c) $\mathrm{Ni}$, and (d) $\mathrm{Au}$. In this simulation, $24 \mathrm{M}_{13}$ clusters were gently collided with one SWCNT. To shorten the calculation duration, the pressure assumed in this study was much higher than that encountered under practical conditions. Hence, the annealing process was carefully monitored to ensure that clusters did not follow each other in a too rapid succession that might result in some unexpected condition. The impact direction was perpendicular to the axis of the carbon nanotube, and at each impact point, about four clusters collided simultaneously. In the case of titanium or iron clusters (Figs. 6(a), 6(b)), the metal atoms seemed to firmly combine with the carbon atoms. In fact, the metal atoms entered the carbon nanotube, and their strong interaction with the carbon atoms caused a structural deformation of the nanotube. Moreover, the continual impacts that reduced the calculation load (10 ps/layer versus $1-250 \mathrm{~ms} /$ layer in typical experiments) also resulted in unrealistic phenomena. In the case of nickel clusters (Fig. 6(c)), the nickel atoms smoothly 
and seamlessly covered the carbon nanotube. The equilibrium position of the nickel atoms coincided with the center of the hexagonal carbon network. When the first nickel cluster collided with the carbon nanotube, a granular structure was temporarily formed. However, the nickel atoms immediately found a stable position and moved into it. Once they reached their equilibrium position, they remained stationary at this temperature. When the second nickel cluster reached the surface, the nickel atoms instantly acquired a granular structure and then moved into the most stable sites. When all the clusters had reached the surface, the equilibrium positions on the carbon network were saturated, and consequently, the nickel clusters had formed a second metal layer on the carbon nanotube. Even at this stage, the nickel atoms did not form a granular structure but rather formed a smooth and seamless layer.

Figure 6(d) shows the result of coating for gold clusters. According to Zhang et al., gold atoms form a highly discontinuous coating with a granular structure. According to our simulation, however, gold atoms can be deposited smoothly but discontinuously. The gold atoms initially formed a granular structure, as reported by Zhang et al., but this was because the gold clusters were accidentally concentrated in the same area on the carbon nanotube. This indicates that if the evaporation rate is decreased or the experiment is conducted under a higher vacuum condition, the formation of a granular structure can be avoided. Zhang et al. explained this result in terms of the weak interaction between the gold atoms and the SWCNT. However, according to our DFT calculations, the binding energy of the Au-C bond is slightly larger than that of the Au-Au bond. The actual value of either binding energy is not considered to be significant; however, the ratio of the binding energies (i.e., $\varepsilon_{\mathrm{M}-\mathrm{C}} / \varepsilon_{\mathrm{M}-\mathrm{M}}$ ) should ideally be substantial. Because each atom has many coordination numbers under the relevant conditions, the approximate values of this ratio for titanium, iron, nickel, and gold are $2,3,1.5$, and 1 , respectively. Thus, in the case of titanium or iron coating, the carbon atoms experience an outward pull and the nanotube structure is deformed. In the case of nickel, a very smooth coating is obtained. On the other hand, in the case of gold, even though the Au-C interaction is slightly stronger than the Au-Au interaction, a granular structure is formed. In some areas, the gold atoms are deposited smoothly but not seamlessly. The reason for this phenomenon is associated with the equilibrium bond length. As mentioned above, the most stable site for the first layer of metal atoms is the center of

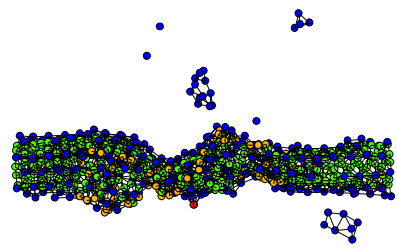

(a) Titanium

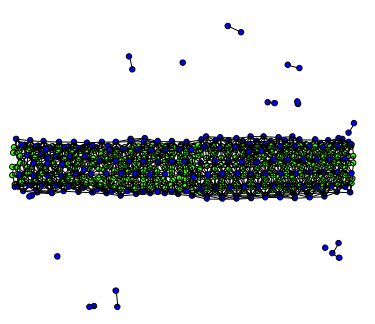

(c) Nickel
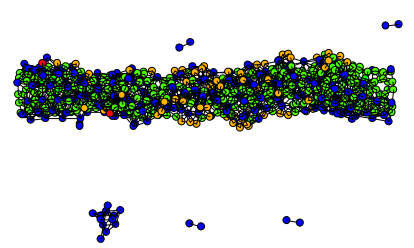

(b) Iron

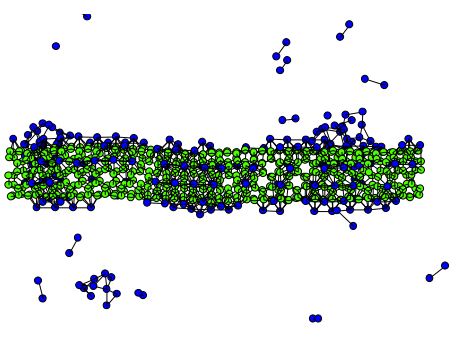

(d) Gold

Fig. 6. Continuous metal evaporation. Blue is metal atom, green is carbon atom with three bonds (sp2), orange is carbon atom with two bonds (one dangling bond), and red is carbon atom with one bond (two dangling bonds). 
the hexagonal carbon network, which has a diameter of $2.5 \AA$. Coincidently, the equilibrium bond length of nickel or iron is comparable, however, that of gold is slightly greater (2.6-3.3 $\AA$, depending on the coordination number). The gold atoms require a shorter bond length so that they can get accommodated by the stable sites of the carbon nanotube, thereby creating a discontinuous coating that reduces the distortion energy. Thus, even gold atoms can be deposited smoothly, provided the distances between them are sufficient to reduce the distortion for each fragment of coating. (In our simulation, each fragment of coating is approximately $10-15 \mathrm{~nm}$ in length). Owing to the strong $\mathrm{C}-\mathrm{Ti}$ and $\mathrm{C}-\mathrm{Fe}$ interactions, the structure of the carbon nanotube was apparently distorted by the high coating rate in our simulation. In order to confirm this, a lower coating rate was applied and the results were examined. In this case, each $\mathrm{M}_{13}$ cluster collides with the center of the carbon nanotube in a sequence. For complete annealing, this sequential impact needs a sufficiently large interval. Figure 7 shows the sequential impact of the metal clusters on the carbon nanotube. A sufficient duration for each impact step results in a coating that is smooth and continuous, with no defects observed in the carbon nanotube. Similar to the nickel atom, the iron atom finds a stable position and moves into it. However, in this simulation, because each cluster reaches almost the same position, the iron atoms do not spread all over the surface but instead form a second layer. This can occur in the nickel system, as well. When too many clusters arrive at the same point, each atom seeks the stable site and tends to move into it; however, the temperature is reduced before diffusion is completed. As a result, the iron atoms form a second layer.

A similar behavior is observed for titanium coating, as shown in Fig. 7(b). However, in the case of titanium clusters, when the impact energy is small, a different phenomenon is

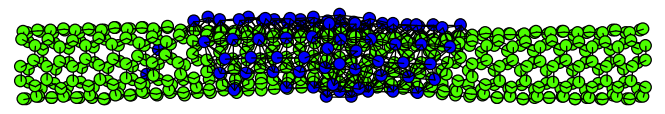

(a) Sequential Fe coating. Totally six Fe13 clusters collide.

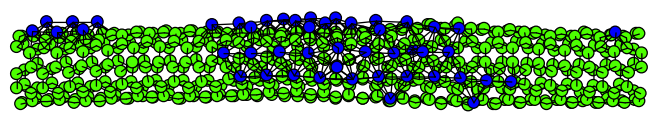

(b) Sequential Ti coating. Totally three Ti13 clusters collide.

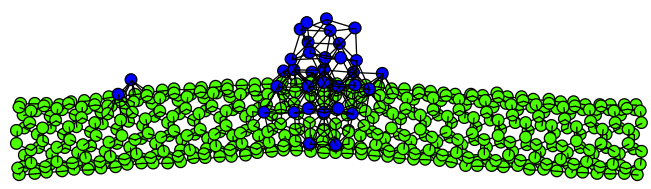

(c) Sequential Au coating. Totally three Au13 clusters collide.

Fig. 7. Sequential impacts of Fe, Ti, and Au cluster on the carbon nanotube surroundings with enough annealing duration after every impact. 
observed. In our simulation, the first three clusters were able to reach the surface, even at a low evaporation energy; however, the next cluster was not able to reach the surface. This can be attributed to the difference in the equilibrium bond lengths. According to Eq. 6, if the number of coordinates is sufficient, the equilibrium bond length must equal $\mathrm{R}_{\mathrm{e} 1}$ for the metal-metal potential, while the metal-carbon equilibrium bond length is simply determined as a function of $R_{e}$ (Table 1). A comparison reveals that the difference in this value is larger for the titanium coating system than for the iron coating system. This is because, when there is already a titanium layer on the carbon nanotube, a cluster with small impact energy cannot overcome the repulsive force. Thus, the cluster is reflected before it can be attracted by the carbon atoms. This proves that at high evaporation energy (e.g., $1 \mathrm{eV}$ ), the titanium clusters can arrive at the surface and produce a smooth coating. Moreover, this result indicates the presence of a reaction barrier. In this simulation, we did not intentionally apply the reaction barrier that is generally applied when using the LJ potential between clusters and an SWCNT, because the energy barrier has not been cleared at the moment. However, because we employed the many-body potential for metal-metal and metal-carbon interactions, taking into account the coordination number, a reaction barrier can be observed in some cases, depending on the cluster environment. In the case of gold coating, as shown in Fig. 6(c), the first cluster was able to spread over the surface; however, the second and the third clusters were not able to spread over the surface and therefore formed a granular structure. This indicates that if gold clusters reach the same place, as in the experiment with a high deposition rate, granular formation is unavoidable, as can be inferred from Fig. 7(d). As mentioned in the section above, the contact angle is closely related to wettability, which is well explained by solid-liquid affinity $\left(\varepsilon_{\mathrm{s} l} / \varepsilon_{\mathrm{l}}\right)$. According to their results, the contact angle tends to increase as the solid-liquid affinity decreases, indicating that the gold clusters tend to form a granular structure. Through these simulations, atomic deposition and surface migration were evident; however, spattering phenomena and infusion were not confirmed, so the evaporation energy used in this simulation is considered to be quite appropriate.

\subsection{Physical properties of functionalized SWCNT}

Figure 8 shows the stress-strain (force-strain) curves of the coated and the uncoated SWCNTs. In this simulation, one end of the SWCNT was fixed while the other end was pulled slowly enough to permit annealing and prevent unintentional breaking of SWCNT. A sufficient relaxation time of $10 \mathrm{ps}$ for each stretch of $0.1 \AA$ was estimated from the vibrational decay. Owing to the disturbance of the perfect $s p^{2}$ bonding in the SWCNT, it might well be expected to become much weaker. However, its physical strength is not greatly reduced, or rather, the force constant becomes larger. Rupture is induced by metal atoms that tend to steal the bonds from the carbon. Because the bond length of carbon-metal is longer than that of carbon-carbon, the interaction between the carbon atoms suddenly becomes weaker than that of carbon with metal when the SWCNT is pulled. With respect to reproducibility, because the yield stress of the functionalized SWCNT has not been reported, it is difficult to confirm its accuracy. However, the equilibrium position of the metal is the center of the hexagonal carbon network, which is quite symmetric. This condition is similar to that of deriving the bond energy by using DFT calculations, thus we consider the result to be qualitatively correct. On the other hand, with regard to the quantitative accuracy, the yield stress and Young's modulus derived from the Brenner potential can vary by several times $^{(22,23)}$..

Figure 9 shows the thermal diffusivities of coated and uncoated SWCNTs. The thermal diffusivity is obtained by fitting the following one-dimensional non-Fourier heat conduction equation with two relaxation timescales $\left(\tau_{1}, \tau_{2}\right)$ : 


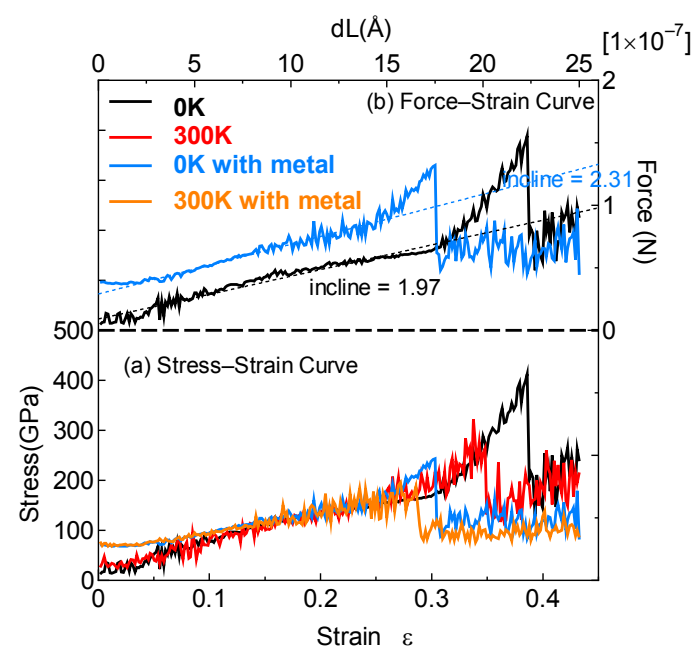

Fig. 8. The stressstrain curve (a) and forcéstrain curve (b). The stress is defined by assuming their cross section with $6.93 \AA$ for the uncoated SWCNT and $8.32 \AA$ for the metal-coated SWCNT in diameter. The metal-coated SWCNT meets earlier rupture point but has a larger force constant. The metal-coated SWCNT has a residual stress that makes the inclination flat in a small strain (displacement) range.

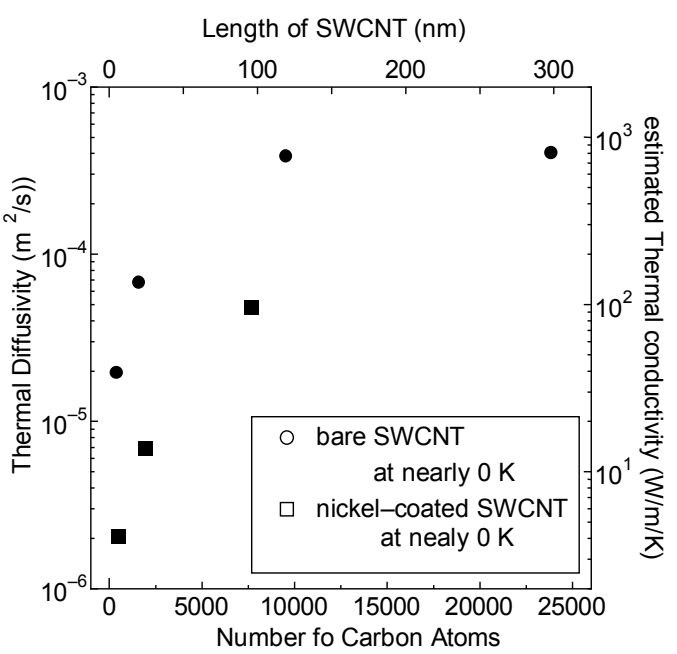

Fig. 9. Thermal diffusivity estimated in this study. The thermal diffusivity of the nickel-coated SWCNT decreases by $90 \%$. The estimated thermal conductivity $(\lambda)$ is expressed as follows: $\lambda=1 \times 10^{6} \alpha-3 \times 10^{6} \alpha$. It depends on the definition of the density and specific heat capacity $\left(\mathrm{C}_{\mathrm{v}}\right)$ of the SWCNTs is assumed to be $3 \mathrm{R}$, where R is a gas constant.

$\tau_{1} \frac{\partial^{2} T}{\partial t^{2}}+\frac{\partial T}{\partial t}=\alpha\left(\nabla^{2} T+\tau_{2} \frac{\partial}{\partial t} \nabla^{2} T\right)$

The dominant heat carrier in the SWCNT is the phonon, and this model is fully described in the literature ${ }^{(24)}$. As shown in Fig. 9, the thermal conductivity is drastically decreased by coating with nickel atoms. It is well known that when the phonon mean free path $^{(25)}$ is long enough in comparison with the length of the SWCNT, the heat conduction exhibits ballistic behavior. Therefore, a dependence on size was expected only for an uncoated SWCNT of smaller length. However, in this simulation, the size effect is still exhibited. This indicates that even for the metal-coated SWCNT, the phonon mean free path is longer than the calculated range. Thus, the reduction in thermal conductivity is 
presumably due to phonon dispersion. Finally, the thermal conductivity of the bare SWCNT is found similar to that given in the paper mentioned above and is required to reproduce the experimental value to calculate a greater length. However, the result for the functionalized SWCNT is one greatly worth having. Although this phenomenon is derived from newly built potential parameters, the condition of the equilibrium structure is quite similar to the condition of deriving the parameters, so that it is considered appropriate. Because at present there are no similar reports, further experimental and numerical studies will make matters clearer.

\section{Conclusion}

In this paper, the MD approach for CNT analysis was explained. The classical MD merely solves the Newtonian equation; however, if the potential function is fully appropriate, the results must be credible. In the case of PVD and coating, the simulation showed good agreement with the experimental results and the deposition mechanism became clear. In the case of a metal-coated SWCNT, the physical strength was comparable with that of the uncoated SWCNT; however, its thermal conductivity decreased drastically. In this study, the size effect of thermal conductivity was not shown, even for a metal-coated SWCNT, which indicates that the reduction in thermal conductivity is not caused by phonon dispersion.

\section{References}

(1) Iijima, S., Helical microtubeles of graphitic carbon. Nature, Vol. 35 (199), pp. 56-58

(2) Iijima, S., Ichihashi, T., Single-shell carbon nanotubes of 1-nm diameter. Nature, Vol.363, No.6430 (1993), pp.603-605.

(3) Nygard, J., Cobden, D.H., Lindelof, P.E., Kondo physics in carbon nanotubes. Nature, Vol.408, No.6810 (2000), pp.342-346.

(4) Derycke, V., Martel, R., Appenzeller, J., Avouris, P., Carbon nanotube inter- and intramolecular logic gates. Nano Letters, Vol.1, No.9 (2001), pp.453-456.

(5) Wong, S.S., Joselevich, E., Woolley, A.T., Cheung, C.L., Lieber, C.M., Covalently functionalized nanotubes as nanometre-sized probes in chemistry and biology. Nature, Vol.394, No.6688 (1998), pp.52-55.

(6) Wu, Z.C., Chen, Z.H., Du, X., Logan, J.M., Sippel, J., Nikolou, M., Kamaras, K., Reynolds, J.R., Tanner, D.B., Hebard, A.F., Rinzler, A.G., Transparent, conductive carbon nanotube films. Science, Vol.305, No.5688 (2004), pp.1273-1276.

(7) Wang QY, Johnson JK., Optimization of carbon nanotube arrays for hydrogen adsorption. J. Phys. Chem. B, Vol.103, No.23 (1999), pp.4809-4813.

(8) Hertel T, Martel R, Avouris P., Manipulation of individual carbon nanotubes and their interaction with surfaces. J. Phys. Chem. B, Vol.102, No.6 (1998), pp.910-915.

(9) Yu, R.Q., Chen, L.W., Liu, Q.P., Lin, J.Y., Tan, K.L., Ng, S.C., Chan, H.S.O., Xu, G.Q., Hor, T.S.A., Chem. Mater., Vol.10, No.3 (1998), pp.718-722.

(10) Liu, J., Casavant, M.J., Cox, M., Walters, D.A., Boul, P., Lu, W., Rimberg, A.J., Smith, K.A., Colbert, D.T., Smalley, R.E., Controlled deposition of individual single-walled carbon nanotubes on chemically functionalized templates. Chem. Phys. Lett., Vol.303, No.1-2 (1999), pp.125-129.

(11) Duong, H.M., Ishikawa, K., Okawa, J., Ogura, K., Einarsson, E, Shiomi, J., Maruyama, S., Mechanism and optimization of metal deposition onto vertically aligned single walled carbon nanotube arrays. J. Phys. Chem. C, vol. 113 (2009), pp. 14230-14235.

(12) Zhang, Y., Franklin, N.W., Chen, R.J., Dai, H.J., Metal coating on suspended carbon nanotubes and its implication to metal-tube interaction. Chem. Phys. Lett., Vol.331, No.1 
(2000), pp.35-41.

(13) Yamaguchi, Y., Maruyama, S., A molecular dynamics simulation of the fullerene formation process. Chem. Phys. Lett., Vol.286, No.3-4 (1998), pp.336-342.

(14) Finnis, M.W., Sinclair, J.E., A Simple Empirical N-Body Potential for Transition-Metals. Philos. Mag.A, Vol.50, No.1 (1984), pp.45-55.

(15) Shibuta, Y., Maruyama, S., Bond-order potential for transition metal carbide cluster for the growth simulation of a single-walled carbon nanotube. Comput. Mat. Sci., Vol.39, No.4 (2007), pp.842-848.

(16) Becke, A.D., Density-Functional Thermochemistry .3. The Role of Exact Exchange. J. Chem. Phys., Vol.98, No.7 (1993), pp. 5648-5652.

(17) Lee, C.T., Yang, W.T., Parr, R.G., Development of The Colle-Salvetti Correlation-Energy Formula into a Functional of The Electron-Density. Phys. Rev. B, Vol.37, No.2 (1988), pp. 785-789.

(18) Hay, P.J., Wadt, W.R., Abinitio Effective Core Potentials for Molecular Calculations Potentials for the Transition-Metal Atoms Sc to Hg. J. Chem. Phys., Vol.82, No.1 (1985), pp. 270-283.

(19) Castro, M., Jamorski, C., Salahub, D.R., Structure, bonding, and magnetism of small Fe-n, Co-n, and Ni-n, clusters, $\mathrm{n}<=5$. Chem. Phys. Lett., Vol.271 (1997), pp. 133-42.

(20) Morse, P.M., Diatomic Molecules According to the Wave Mechanics. II. Vibrational Levels. Phys. Rev., Vol.34 (1929), pp. 57-64.

(21) Maruyama, S., Kurashige, T., Matsumoto, S., Yamaguchi, Y. Kimura, T., Liquid Droplet in Contact with a Solid Surface. Micro. Thermophys. Eng., Vol.2, No.1 (1998), pp. 49-62.

(22) Yakobson, B.I., Brabec, C.J., Bernholc, J., Nanomechanics of carbon tubes: Instabilities beyond linear response. Phys. Rev. Lett., Vol.76, No.14 (1996), pp.2511-2514.

(23) Xie, S.S., Li, W.Z., Pan, Z.W., Chang, B.H., Sun, L.F., Mechanical and physical properties on carbon nanotube. J. Phys. Chem. Solids, Vol.61, No.7 (2000), pp.1153-1158.

(24) Shiomi, J., Maruyama, S., Non-Fourier heat conduction in a single-walled carbon nanotube: Classical molecular dynamics simulations. Phys. Rev. B, vol.73 (2006), pp. 205420-1-205420-7.

(25) Dresselhaus, M.S., Eklund, P.C., Phonons in carbon nanotubes. Adv. Phys., Vol.49, No.6 (2000), pp.705-814.

(26) Hone, J., Whitney, M., Piskoti, C., Zettl, A., Thermal conductivity of single-walled carbon nanotubes. Phys. Rev. B, Vol.59, No.4 (1999), pp. 2514-2516.

(27) Berber, S., Kwon, Y.K., Tomanek, D., Unusually high thermal conductivity of carbon nanotubes. Phys. Rev. Lett., Vol.84, No.20 (2000), pp.4613-4616. 